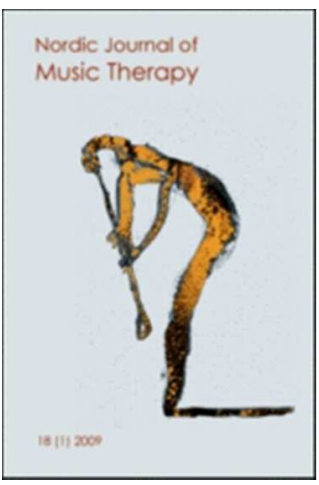

Music therapy and cognitive capacity in people with Alzheimer's disease:

A call for action

\begin{tabular}{|r|l|}
\hline Journal: & Nordic Journal of Music Therapy \\
\hline Manuscript ID: & RNJM-2013-0032.R2 \\
\hline Manuscript Type: & Letter to the Editor \\
\hline & \\
\end{tabular}

SCHOLARONE ${ }^{m}$

Manuscripts 


\title{
Music therapy and cognitive capacity in people with Alzheimer's disease: A call for action
}

\begin{abstract}
Alzheimer's disease (AD) is the most common type of dementia, affecting memory, thinking, behaviour and the ability to perform activities of daily living. The increase in life expectancy worldwide is expected to translate into many more cases of dementia. In fact, Alzheimer's Disease International (2009) estimated that in 2010 there were 36 million people living with dementia worldwide, and this number is expected to reach 115 million by 2050 .
\end{abstract}

Although pharmacological interventions appear to slow AD symptoms progression, no effective long-term treatment has been established and symptoms typically worsen progressively. The current limits on the effectiveness of drugs, their adverse effects and the requirement for a range of options are highlighting the need for robust evaluations of non-pharmacological therapeutic interventions in AD.

Our professional experience tells us that the use of music or its elements (sound, rhythm, melody and harmony) has an impact on cognitive function in people with AD. In order to check this hypothesis, we screened from January 2000 to June 2013 the electronic database PubMed for music intervention studies with people with AD in which cognitive function was one of the selected outcomes. The terms used in different combinations were; Alzheimer, Alzheimer's disease, dementia, music, and music therapy. Inclusion criteria were: (1) intervention study; (2) based on music; (3) with people diagnosed with $\mathrm{AD}$; (4) and cognitive capacity measured as outcome. From the 251 initially selected papers, 244 were refused after reading title and/or abstract. After reading the full text only 2 studies meet our four basic inclusion criteria (Brotons and Koger 2000; Boulay et al. 2011). 
Obviously, there is a lack of systematic testing or randomized controlled trials analyzing the effects of music therapy on cognitive capacity in people with AD. There is only partial understanding of the processes underlying the interventions and results of music therapy on people with dementia. In fact, there is a small number of music-based interventions focused on cognitive capacity in people with $\mathrm{AD}$, and with crucial methodology limitations (Vink et al. 2011).

In our opinion, further intervention studies with people with AD aimed to the check the potential use of music-therapy stopping cognitive degeneration or even improving cognition should be performed. Below, we have identified some key areas which we believe are of importance in developing new research in this field.

- There is no single test that proves a person has AD, but a high number of new diagnostic tools are emerging. Future research should use more precise diagnosis tools for screening $\mathrm{AD}$ in early stages such as analyzing plasma levels of amyloid- $\beta$.

- The Mini-Mental State Examination (MMSE) is the most commonly administered assessment tool for the diagnosis and the monitoring of dementia. However, some evidences indicate that MMSE may not be adequately sensitive to track disease progression. Future research should assess cognitive capacity by more specific and sensitive tools such as magnetic resonance imaging of the brain.

- Some cognitive aspects such as spatial cognition, short term memory, language functioning or attention have been studied in relation to music, but the effects of music on the brain and at a cellular level have not been clarified yet. We firmly believe that music can be a positive stimulus for many cognitive aspects in 
people with AD. Future research should evaluate the efficacy of diverse musical activities (e.g. listening, singing, playing instruments, etc.) on different cognitive aspects, even checking a possible dose-effect relationship.

\begin{abstract}
Although AD presents challenges unresolved to date, this underexplored field of research seems promising. We hope that this letter will provide a platform for stimulating advances as well as a rationale for immediate improvements in music therapy interventions with people with AD.
\end{abstract}

\title{
References
}

Alzheimer's Disease International (2009). World Alzheimer Report 2009. A.s.D. International. London.

Boulay, M., Benveniste, S., Boespglug, S., Jouvelot, P., and Rigaud, A.S. (2011). "A pilot usability study of MINWii, a music therapy game for demented patients." Technology and health care: official journal of the European Society for Engineering and Medicine 19(4): 233-46.

Brotons, M., and Koger, S.M. (2000). "The impact of music therapy on language functioning in dementia." Journal of music therapy 37(3): 183-95.

Vink A.C., Bruinsma M.S., and Scholten R.J.P.M. (2011). Music therapy for people with dementia. Cochrane Database of Systematic Reviews. CD003477. 